\title{
Long-term model of colitis-associated colorectal cancer suggests tumor spread mechanism and nature of cancer stem cells
}

\author{
YUJI TANIMURA, TOSHIRO FUKUI, SHUNSUKE HORITANI, YASUSHI MATSUMOTO, SACHI MIYAMOTO, \\ RYO SUZUKI, TOSHIHIRO TANAKA, TAKASHI TOMIYAMA, TSUKASA IKEURA, \\ YUGO ANDO, AKIYOSHI NISHIO and KAZUICHI OKAZAKI
}

Third Department of Internal Medicine, Division of Gastroenterology and Hepatology, Kansai Medical University, Hirakata, Osaka 573-1010, Japan

Received March 30, 2020; Accepted October 12, 2020

DOI: $10.3892 / 01.2020 .12268$

\begin{abstract}
Although chemical-induced animal models of colorectal cancer (CRC) suggest a lot about the disease, more efforts are required to establish metastasis models. Azoxymethane (AOM) and dextran sodium sulfate (DSS)-treated (AOM/DSS) Crl:CD-1 mice were sacrificed after 10 or 20 weeks in our previous study, and most colon tumors exhibited intramucosal adenocarcinomas. Our observations were extended until 30 weeks to study a colitis-associated advanced CRC mouse model, and explore whether linker threonine-phosphorylated Smad2/3 (pSmad2/3L-Thr) immunostaining-positive cells were involved in the progressive course of colitis-associated CRC as cancer stem cells. AOM/DSS mice were sacrificed at 10, 20 and 30 weeks after AOM administration. Following the histopathological analysis, immunohistochemical staining was performed for the following markers: CD34, podoplanin, $\beta$-catenin, E-cadherin, Ki67, Bmil and pSmad2/3L-Thr. Compared with AOM/DSS mice at 10 and 20 weeks, submucosal tumor infiltration and tumor invasion into vessels were markedly increased at 30 weeks. In the parts of colon tumors
\end{abstract}

Correspondence to: Dr Toshiro Fukui, Third Department of Internal Medicine, Division of Gastroenterology and Hepatology, Kansai Medical University, 2-5-1 Shinmachi, Hirakata, Osaka 573-1010, Japan E-mail: fukuitos@hirakata.kmu.ac.jp

Abbreviations: UC, ulcerative colitis; CRC, colorectal cancer; AOM, azoxymethane; DSS, dextran sodium sulfate; EMT, epithelial-mesenchymal transition; CSC, cancer stem cell; TGF- $\beta$, transforming growth factor- $\beta$; MH, Mad homology; T $\beta$ RI, TGF- $\beta$ type I receptor; Ser, serine; Thr, threonine; ERK, extracellular signal-regulated kinase; JNK, c-Jun NH2-terminal kinase; CDK, cyclin-dependent kinase; pSmad2/3L-Thr, linker threonine-phosphorylated Smad2/3; ICR, Crl:CD-1; H\&E, hematoxylin and eosin; TBS, Tris-buffered saline; Abs, antibodies; Bmi1, B cell-specific Moloney murine leukemia virus integration site 1; DAPI, 4',6-diamidino-2-phenylindole; SEM, standard error of the mean

Key words: mouse model, colitis-associated colorectal cancer, metastasis, cancer stem cell, Smad, epithelial-mesenchymal transition from AOM/DSS mice, particularly in mice at 30 weeks, the positive signal of E-cadherin was clearly reduced in the cell membranes. The percentage of Ki67-positive tumor cells in mucosal areas of AOM/DSS mice was higher than that in the sites of submucosal infiltration. In mucosal areas of colon tumors, pSmad2/3L-Thr-positive cells were scattered among tumor cells. At sites of submucosal infiltration and vessel invasion of these tumors, pSmad2/3L-Thr-positive cells were also observed among tumor cells. In colon tumors from AOM/DSS mice at 30 weeks, the percentage of pSmad2/3L-Thr-positive cells among the nuclear $\beta$-catenin-positive tumor cells was higher than that among the cytoplasmic $\beta$-catenin-positive tumor cells. For both non-neoplastic and neoplastic epithelial cells, pSmad2/3L-Thr-positive cells exhibited immunohistochemical co-localization with Bmi1. The present study developed an advanced CRC mouse model that exhibited tumor infiltration into the submucosa and invasion into vessels. The present study re-confirmed the theory that pSmad2/3L-Thr-positive cells may be cancer stem cells.

\section{Introduction}

Ulcerative colitis (UC) poses a high risk of developing colorectal cancer (CRC) (1), and the number of patients with $\mathrm{UC}$-associated CRC is increasing all over the world as that with $\mathrm{UC}$ increases (2). The risk of developing UC-associated CRC is affected by the degree and duration of inflammation in UC and genetic predisposition (3). However, the detailed molecular mechanism underlying transition from UC-associated inflammation to carcinogenesis has not yet been elucidated.

Some animal models of sporadic and colitis-associated CRC have been developed in rodents. Induction of the most well-studied mouse model of chemical-induced colitis-associated CRC requires a single intraperitoneal injection of azoxymethane (AOM), a carcinogen of the colon, followed by colitis induction through oral administration of dextran sodium sulfate (DSS) (4,5). This AOM/DSS mouse model reproduces the course of human colitis-associated CRC from inflammation to dysplasia and carcinoma, producing severe colitis with weight loss, bloody diarrhea, and multiple colon tumors $(5,6)$.

There are other aspects to the mouse models of CRC, including the AOM/DSS model. There are significant 
differences in invasive and metastatic potentials of CRC between animal models and human disease (6-8). At diagnosis, approximately $50 \%$ of CRC patients have lymphatic metastases and $33 \%$ have hematogenous metastases (6). In contrast, the frequency of metastases is extremely low in AOM models of CRC (8). In human CRC, the disease progresses in order, and a usual route of hematogenous metastasis first reaches the liver, and subsequently the lung. However, metastatic liver tumors are rare in the AOM models of rodent CRC. Although animal models of chemical-induced CRCs have provided much information about human disease, many research approaches have not been made available, and further research to establish animal models of metastasis is needed.

Besides remodeling microenvironment to promote metastasis, cancer cells actuate regulators of embryonic morphogenesis to achieve epithelial-mesenchymal transition (EMT) and stop the differentiation program. Thereby, cancer cells acquire motility, invade, and gain stem-like characters $(9,10)$. Recently, it has been revealed that cancer stem cells (CSCs) and EMT-type cells have common molecular features and play important roles in tumor metastasis (11-13). The development of CSCs and EMT can promote the formation of metastatic tumors in $\mathrm{CRC}$, but the molecular mechanisms of metastasis involving CSCs and EMT remain unclear (14). Furthermore, because CSCs are comparatively resistant to therapies created to eradicate cancer cells with non-CSC characters, analyses of CSCs, which are small in number and are multi-drug resistant, form the bases for the development of new therapeutic agents targeting CSCs, and open the door to new cancer treatments (11).

Smad proteins are core mediators that transmit signals from transforming growth factor (TGF)- $\beta$ superfamily receptors to the nuclei. They are regulatory proteins consisting of conserved Mad homology ( $\mathrm{MH}) 1$, intermediate linker, and MH2 domains $(15,16)$.

TGF- $\beta$ type Ireceptor (T $\beta$ RI) with catalytic activity phosphorylates $\mathrm{COOH}$-terminal serine (Ser) residues of receptor-activated Smads, including Smad2 and Smad3 (17). Certain Ser or threonine (Thr) residues in the linker are phosphorylated by Ras-related (proline-directed) kinases, consisting of extracellular signal-regulated kinase (ERK), c-Jun NH2-terminal kinase (JNK), and cyclin-dependent kinase (CDK)4 (18-21). T $\beta R I$ and Ras-related kinases specifically phosphorylate Smad2 and Smad3, creating several phosphoisoforms: $\operatorname{Smad} 2 / 3$ phosphorylated at the $\mathrm{COOH}$-terminal (pSmad2C and $\mathrm{pSmad} 3 \mathrm{C})$; Smad2/3 phosphorylated at the linker ( $\mathrm{pSmad} 2 \mathrm{~L}$ and $\mathrm{pSmad} 3 \mathrm{~L}$ ); and Smad2/3 phosphorylated at both the C-terminal and linker (pSmad2C/L and pSmad3C/L) (21-24). Phosphorylated Smad2 and Smad3 promptly form oligomers with Smad4 and translocate to the nucleus. There, they control transcription of the target genes (25).

In our previous study, we confirmed specific expression of linker Thr-phosphorylated Smad2/3 (pSmad2/3L-Thr) in mouse colon epithelial cells, and proposed that these cells are colon epithelial stem-like cells (26). Subsequently, we investigated AOM/DSS mouse model, and clarified that carcinogenic pSmad3L-Ser signaling caused by chronic colitis is a significant early event of colitis-associated CRC, by investigating the Smad2/3 phosphorylation profiles. Furthermore, the study has supported the theory that pSmad2/3L-Thr immunostaining-positive cells are CSCs (27). AOM/DSS mice were sacrificed 10 or 20 weeks after AOM administration in that study, and most colon tumors showed the characteristics of intramucosal adenocarcinoma. Around 40 weeks after AOM administration, the number of AOM/DSS mice dying had markedly increased pre-experimentally.

Therefore, in the present study, we extended our observations until 30 weeks after AOM administration to study a colitis-associated advanced CRC mouse model, and explore if pSmad2/3L-Thr immunostaining-positive cells are involved in a progressive course of colitis-associated CRC as CSCs.

\section{Materials and methods}

Mice. We purchased 5-week-old male Crl:CD-1 (ICR) mice from Charles River Laboratories (Charles River Laboratories Japan, Inc.). All mice were kept in the animal facility of Kansai Medical University under specific pathogen-free environment. Mice were fed commercial food pellets (F2; Funabashi Farm) and tap water. All experimental protocols were approved by the Ethics Committee for the Use of Experimental Animals of Kansai Medical University (approval no. 19-006).

Chemicals. We purchased AOM, a colon carcinogen, from Sigma-Aldrich Japan K.K. and DSS having a molecular weight of 36,000-50,000 from MP Biomedicals. DSS was diluted with water to form $2 \%$ solution to induce colitis.

Experimental design. A single intraperitoneal injection of AOM (10 $\mathrm{mg} / \mathrm{kg}$ body weight) was administered to ICR mice, and one week after the AOM administration, the mice were given $2 \%$ DSS in their drinking water for 7 days. Mice administered with AOM/DSS (AOM/DSS mice) were sacrificed by cervical dislocation 10 (week 10; $\mathrm{n}=25$ ), 20 (week 20; $\mathrm{n}=25$ ) or 30 (week 30; $\mathrm{n}=25$ ) weeks after AOM administration (Fig. 1) $(5,27,28)$.

Colons were excised after flushing the lumens with saline and cut open longitudinally. After several washes with saline, they were cut and fixed in 10\%-buffered formalin. Paraffin-embedded sections were prepared by using standard method.

Histopathological analysis. Histopathological changes were confirmed in hematoxylin and eosin (H\&E)-stained specimens. Colorectal neoplasms were diagnosed based on the description of Ward (29). CRC infiltration into the submucosal layer, and invasion into vessels could also be observed in these sections. All cases of CRC invasion into vessels were re-confirmed by immunohistochemical staining of the blood vessels or lymph vessels as explained below in detail.

Domain-specific antibody against the phosphorylated Smad2 and Smad3. Rabbit polyclonal anti-human pSmad2/3L-Thr (Smad2: Thr 220; Smad3: Thr 179) sera were produced against the phosphorylated linker region of Smad2 and Smad3 by immunizing rabbits with synthetic peptides $(23,30,31)$. The antisera were subjected to antigen affinity purification using phosphorylated peptides as previously described (32).

Immunohistochemistry. We performed immunohistochemical staining on formalin-fixed paraffin-embedded sections as 


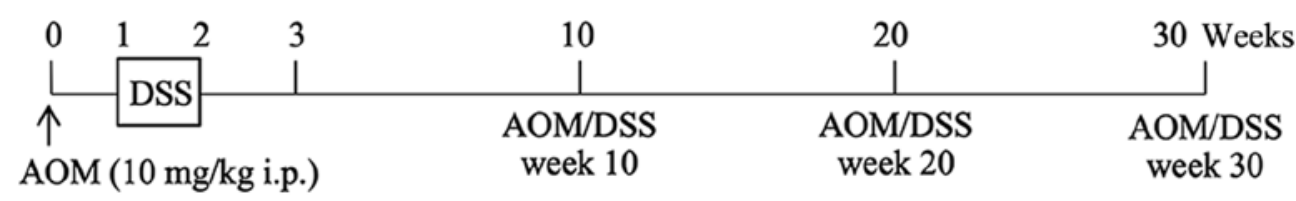

Figure 1. Experimental procedure for establishing a colitis-associated colorectal cancer mouse model. $\uparrow$, AOM, 10 mg/kg body weight, i.p. injection; $\square, 2 \%$ DSS in drinking water; - - basic diet and tap water. AOM, azoxymethane; DSS, dextran sodium sulfate; i.p., intraperitoneal.

described previously $(26,27,33)$. Briefly, paraffin-embedded sections were deparaffinized and rehydrated by washing with xylene and ethanol. Non-enzymatic antigen retrieval was performed by heating sections to $121^{\circ} \mathrm{C}$ for $10 \mathrm{~min}$ in $0.01 \mathrm{M}$ sodium citrate buffer ( $\mathrm{pH}$ 6.0). After cooling, sections were immersed in Tris-buffered saline (TBS) and blocked with 3\% bovine serum albumin dissolved in TBS for $5 \mathrm{~min}$. Primary antibodies (Abs) were diluted with TBS containing $0.1 \%$ Tween-20, and incubated at $4^{\circ} \mathrm{C}$ in a humidity chamber. The primary Abs used in this study were as follows: mouse monoclonal anti-human $\beta$-catenin Ab (sc-7963; Santa Cruz Biotechnology, Inc.), rat monoclonal anti-mouse CD34 Ab (ab8158; Abcam), rat monoclonal anti-mouse podoplanin $\mathrm{Ab}$ (015-24111; Fujifilm Wako Pure Chemical Corp.), rabbit polyclonal anti-human E-cadherin Ab (sc-7870, Santa Cruz Biotechnology, Inc.), rat monoclonal anti-mouse Ki67 Ab (652402; BioLegend), goat polyclonal anti-human B cell-specific Moloney murine leukemia virus integration site 1 (Bmi1) $\mathrm{Ab}$ (ab115251; Abcam), and rabbit polyclonal anti-human pSmad2/3L-Thr Ab. The secondary Abs used were the appropriate species-specific AlexaFluor (488 or 568)-conjugated Abs (Thermo Fisher Scientific, Inc.). Slides were mounted in VECTASHIELD mounting medium containing 4',6-diamidino-2-phenylindole (DAPI) (Vector Laboratories) to stain nuclei. Images were taken and captured using a fluorescence microscope (Olympus). After immunofluorescent staining, the specimen slides were immersed in distilled water and the cover glasses were removed so as not to damage the tissues. After sufficiently immersing them in TBS, H\&E staining was performed by a standard staining procedure. Then, we observed the same sections under a light microscope.

Well-oriented lesions from the base to the surface of CRC were selected for counting immunostaining-positive cells using inForm software (PerkinElmer) according to the manufacturer's instructions.

Statistical analysis. Comparing the presence (positives) or absence (negatives) of CRC infiltration into the submucosal layer and invasion into vessels (grouping variable: 0 , negatives; 1, positives), we analyzed the data with Kruskal-Wallis test followed by Mann-Whitney U test with Bonferroni correction.

Comparing the percentages of immunostaining-positive cells, we expressed the values as mean \pm standard error of the mean (SEM). We analyzed the data with paired t-test.

$\mathrm{P}<0.05$ was considered to indicate a statistically significant difference.

\section{Results}

Microscopic observations. As previously reported, we observed flat, nodular, polypoid, or caterpillar-like colon tumors in the middle and distal colons of all AOM/DSS mice
Table I. Frequency of tumor infiltration into the submucosal layer and invasion into vessels in azoxymethane/dextran sodium sulfate mice.

\begin{tabular}{lcc}
\hline Weeks & $\begin{array}{c}\text { Submucosal } \\
\text { infiltration, positive }\end{array}$ & $\begin{array}{c}\text { Vessel } \\
\text { invasion, positive }\end{array}$ \\
\hline Week 10 & $6 / 25(24 \%)^{\mathrm{a}, \mathrm{b}}$ & $0 / 25(0 \%)^{\mathrm{a}, \mathrm{c}}$ \\
Week 20 & $7 / 25(28 \%)^{\mathrm{b}}$ & $1 / 25(4 \%)^{\mathrm{b}}$ \\
Week 30 & $18 / 25(72 \%)$ & $10 / 25(40 \%)$ \\
\hline
\end{tabular}

${ }^{\mathrm{a}}$ n.s. vs. week $20 ;{ }^{\mathrm{b}} \mathrm{P}<0.01$ vs. week $30 ;{ }^{\mathrm{c}} \mathrm{P}<0.001$ vs. week 30 . Data were analyzed using a Kruskal-Wallis test followed by Mann-Whitney U test with Bonferroni correction. n.s., not significant.

at weeks 10, 20 and 30 (data not shown) $(5,27,28)$. The size and number of colon tumors tended to increase with time after the AOM administration.

Colon tumors of AOM/DSS mice at weeks 10 (Fig. 2A), 20 (Fig. 2B), and 30 (Fig. 2C and D) had the typical appearance of adenocarcinoma. The nuclei were enlarged, round or ovoid, with remarkable nucleoli; nuclear polarity was almost lost; there were a lot of mitoses; and goblet cells had completely disappeared.

Tumor infiltrations into the submucosal layer were occasionally observed in AOM/DSS mice at weeks 10 (24\%: 6/25) and 20 (28\%: 7/25). Alternatively, these findings were frequently observed in AOM/DSS mice at week 30 (72\%: 18/25) (Fig. 2D; arrowhead). Compared with AOM/DSS mice at weeks 10 and 20, submucosal tumor infiltrations of AOM/DSS mice significantly increased at week $30(\mathrm{P}=0.0021$ and $\mathrm{P}=0.0051$, respectively). No significant difference was found between AOM/DSS mice at weeks 10 and $20(\mathrm{P}=0.76)$.

Tumor invasions into vessels were scarcely observed in AOM/DSS mice at weeks $10(0 \%$ : 0/25) and 20 (4\%: 1/25). However, these findings were sometimes observed in AOM/DSS mice at week 30 (40\%: 10/25) (Fig. 2D; arrow). Compared with AOM/DSS mice at weeks 10 and 20, tumor invasions into vessels of AOM/DSS mice significantly increased at week $30(\mathrm{P}=0.0005$ and $\mathrm{P}=0.0024$, respectively). No significant difference was found between AOM/DSS mice at weeks 10 and $20(\mathrm{P}=0.32)$. These results are summarized in Table I.

Double immunofluorescent staining for $\beta$-catenin and markers of blood and lymph vessels at sites of tumor invasions into vessels. After we confirmed tumor invasions into vessels by H\&E-stained sections, we performed double immunofluorescent staining for $\beta$-catenin (green; Fig. 3A, B, D and E) with 

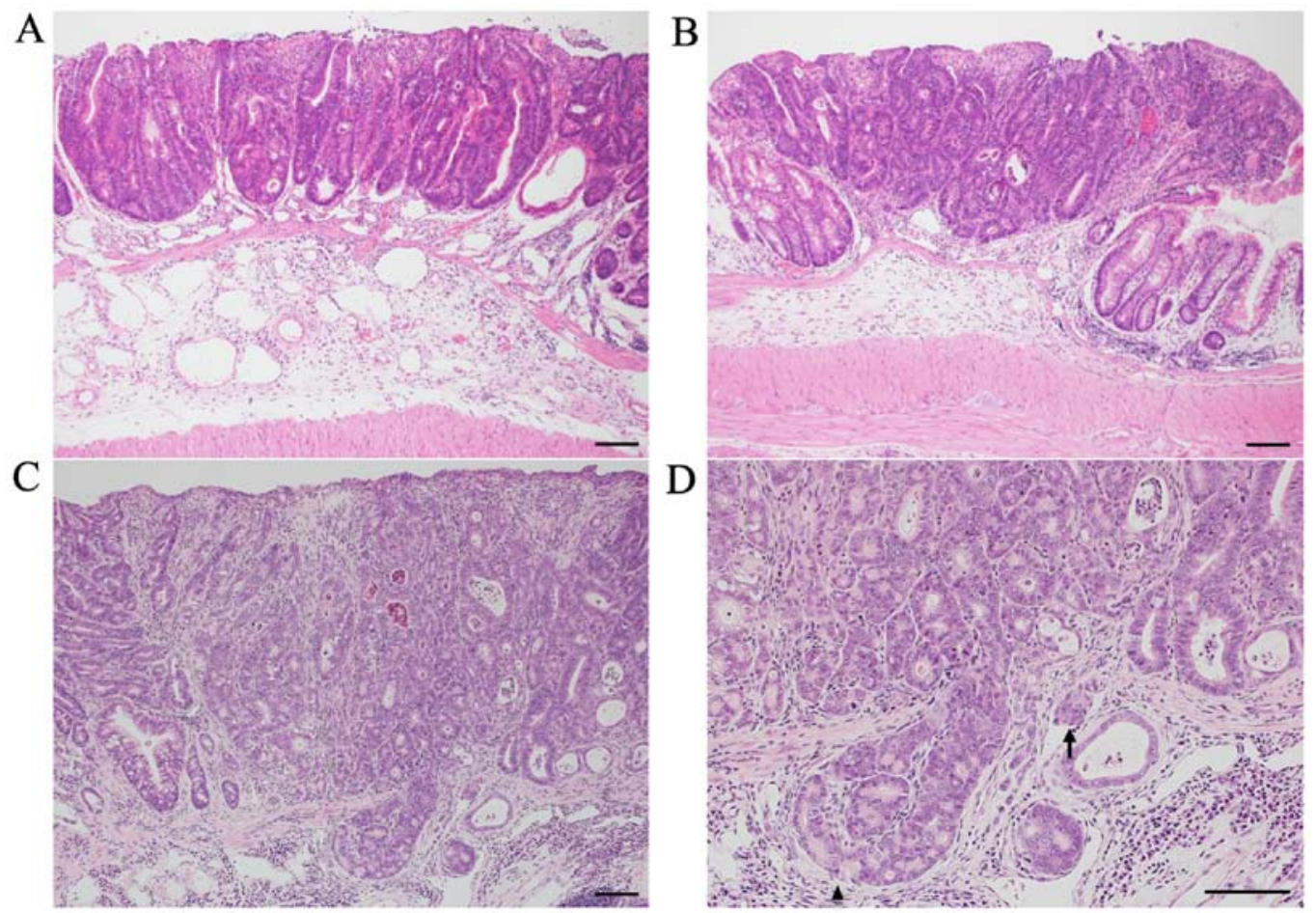

Figure 2. Histopathology of colon tumors from AOM/DSS mice at weeks 10, 20 and 30. Colon tumors of AOM/DSS mice had the typical appearance of adenocarcinoma. Compared with AOM/DSS mice at weeks (A) 10 and (B) 20, (C and D) tumor infiltrations into the submucosal layer (arrowhead) and tumor invasions into vessels (arrow) were more frequently observed in AOM/DSS mice at week 30. Histopathology was performed using hematoxylin and eosin staining. Original magnification, (A-C) x100 and (D) x200. Scale bars, $100 \mu \mathrm{m}$. AOM, azoxymethane; DSS, dextran sodium sulfate.
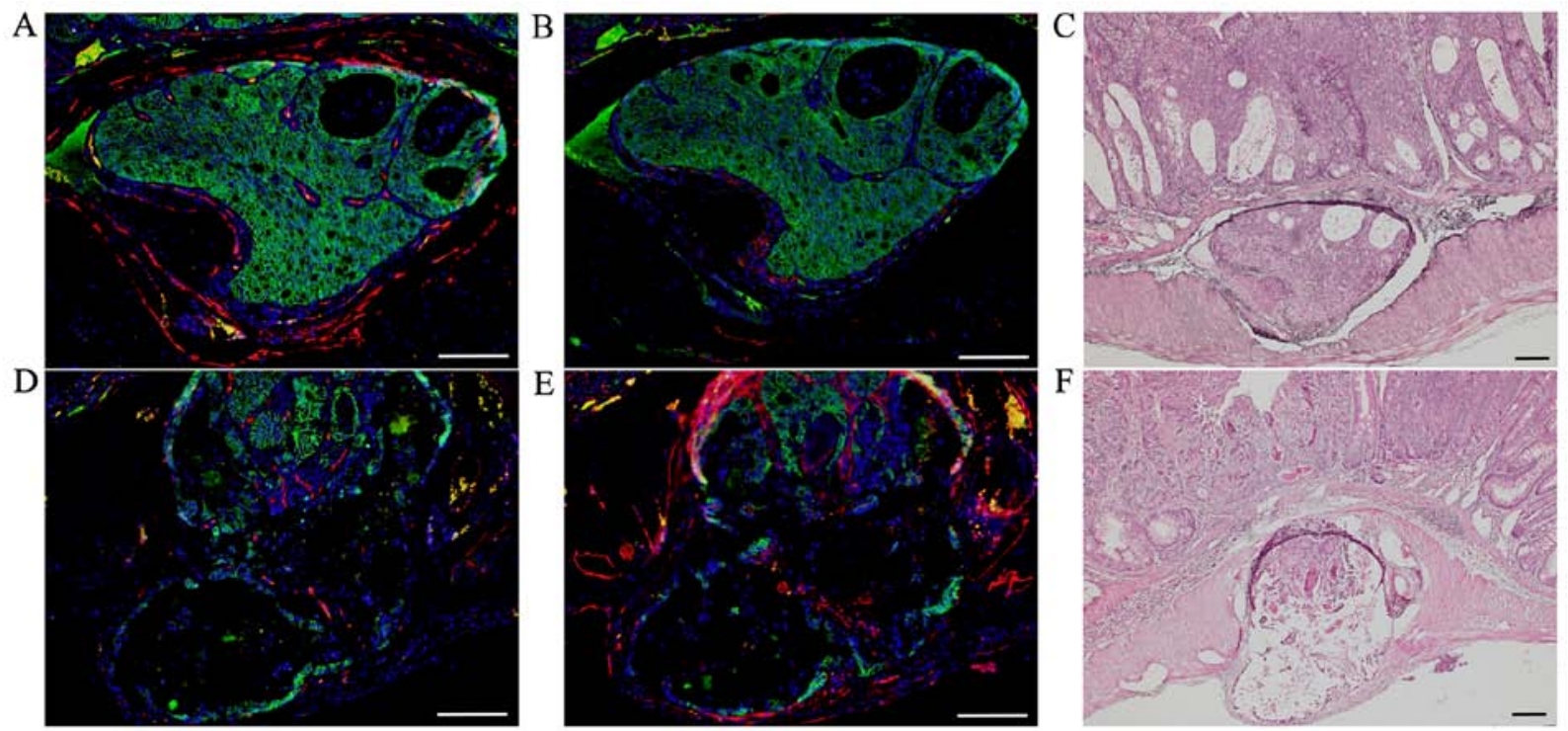

Figure 3. Double immunofluorescent staining of $\beta$-catenin (green) with CD34 (red) and podoplanin (red) in AOM/DSS mice at week 30. DAPI (blue) was used for nuclear staining. (A-C) In AOM/DSS mice that showed blood vessel invasion, (A and B) $\beta$-catenin-positive cells were diffusely distributed throughout the tumors in vessels. (A) Although immunofluorescent staining of CD34 revealed ring-shaped positivity around the vessel lumens, (B) podoplanin-positive cells were not detected in these vessels. (D-F) In AOM/DSS mice that showed lymph vessel invasion, (D and E) $\beta$-catenin-positive tumor cells were similarly distributed in vessels. (D) Contrarily, CD34-positive cells were not detected in the vessels, and (E) immunofluorescent staining of podoplanin demonstrated ring-shaped positivity around the vessel lumens. (C and F) Following immunofluorescent staining, the same sections were stained with hematoxylin and eosin, and observed for tumor invasion into vessels by light microscopy. Original magnification, (A, B, D and E) x200 and (C and F) x100. Scale bars, $100 \mu \mathrm{m}$. AOM, azoxymethane; DSS, dextran sodium sulfate.

CD34 (red; Fig. 3A and D) and podoplanin (red; Fig. 3B and E) to distinguish invasions into blood from lymph vessels in these AOM/DSS mice at weeks $20(\mathrm{n}=1)$ and $30(\mathrm{n}=10)$, using DAPI nuclear staining (blue).
In AOM/DSS mice that showed blood vessel invasion, $\beta$-catenin-positive cells were diffusely distributed throughout the tumors in vessels (Fig. 3A and B). Although immunofluorescent staining of CD34 showed ring-shaped positivity around 

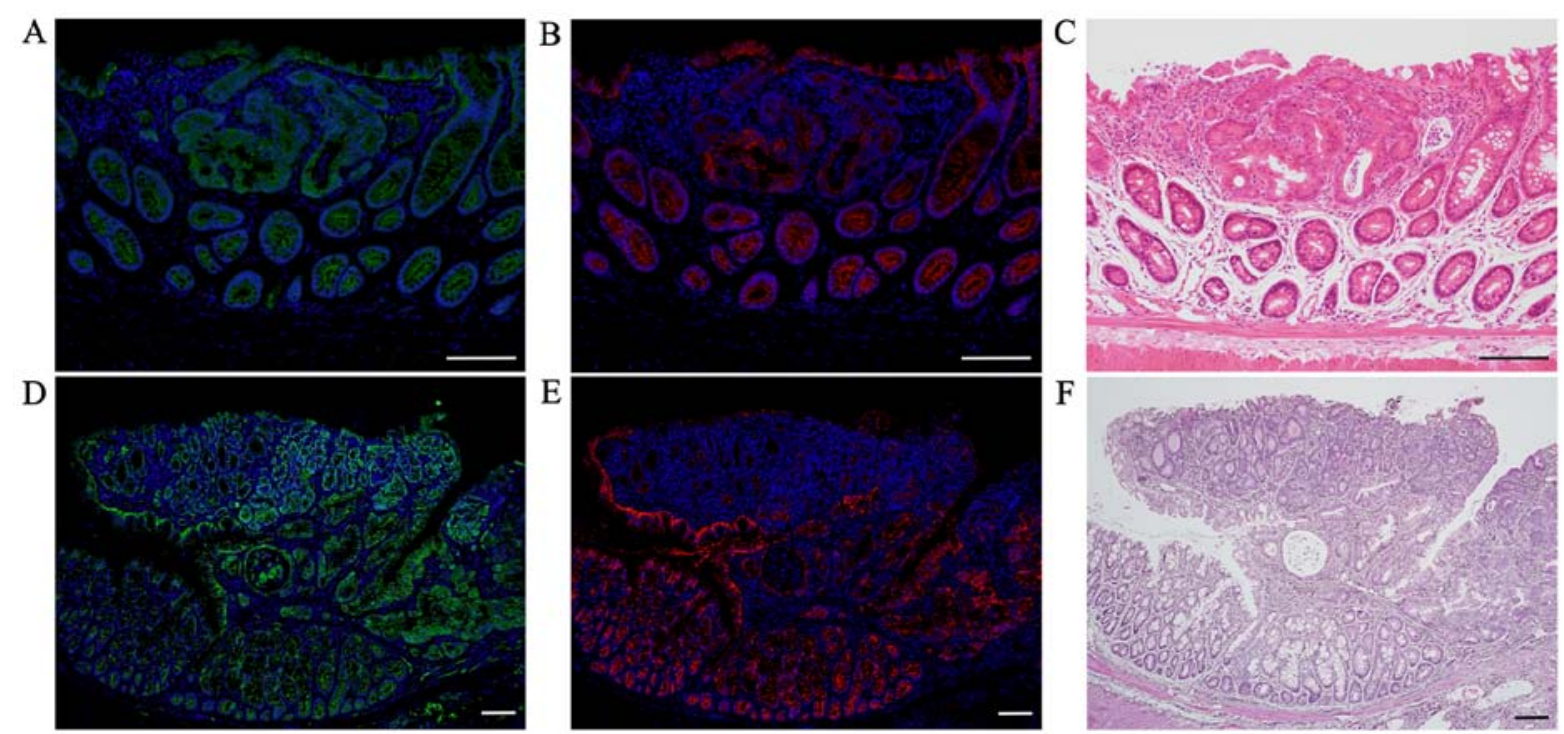

Figure 4. Double immunofluorescent staining for $\beta$-catenin (green) and E-cadherin (red) in AOM/DSS mice at weeks 10 and 30 . DAPI (blue) was used for nuclear staining. Immunofluorescent staining of $\beta$-catenin revealed weak positivity only in the cell membrane of non-tumorous mucosae in AOM/DSS mice at weeks (A) 10 and (D) 30. Strongly $\beta$-catenin-positive cells were distributed throughout the tumors in AOM/DSS mice, and their expression was predominantly observed in the cytoplasm and nucleus of tumor cells. In the same sections, immunofluorescent staining of E-cadherin demonstrated strong positivity in the cell membrane of non-tumorous mucosae in AOM/DSS mice at weeks (B) 10 and (E) 30. Positive levels of E-cadherin in the cell membrane of colon tumors in AOM/DSS mice were clearly reduced as compared with those of non-tumorous mucosae. (C and F) After immunofluorescent staining, the same sections were stained with hematoxylin and eosin, and colon tumors were confirmed using light microscopy. Original magnification, (A-C) x200 and (D-F) x100. Scale bars, $100 \mu \mathrm{m}$. AOM, azoxymethane; DSS, dextran sodium sulfate.

the vessel lumens (Fig. 3A), podoplanin-positive cells were not detected in these vessels (Fig. 3B). In AOM/DSS mice that showed lymph vessel invasion, $\beta$-catenin-positive tumor cells were similarly distributed in vessels (Fig. 3D and E). In contrast, CD34-positive cells were not detected in the vessels (Fig. 3D), and immunofluorescent staining of podoplanin showed ring-shaped positivity around the vessel lumens (Fig. 3E).

Of the $11 \mathrm{AOM} / \mathrm{DSS}$ mice with vessel invasion, tumor invasions into blood and lymph vessels were observed in 3 and 8 mice, respectively.

After immunofluorescent staining, the same sections were stained with H\&E and observed for tumor invasions into vessels under a light microscope (Fig. 3C and F).

Double immunofluorescent staining for $\beta$-catenin and E-cadherin. Immunofluorescent staining of $\beta$-catenin (green; Fig. 4A and D) showed weak positivity only in the cell membrane of non-tumorous mucosae in AOM/DSS mice at weeks 10 (Fig. 4A) and 30 (Fig. 4D), using DAPI nuclear staining (blue). Strongly $\beta$-catenin-positive cells were distributed throughout the tumors in AOM/DSS mice, and their expression was predominantly observed in the cytoplasm and nucleus of tumor cells. In the same sections, immunofluorescent staining of E-cadherin (red; Fig. 4B and E) showed strong positivity in the cell membrane of non-tumorous mucosae in AOM/DSS mice at weeks 10 (Fig. 4B) and 30 (Fig. 4E). Positive levels of E-cadherin in the cell membrane of colon tumors in AOM/DSS mice, particularly in mice at week 30 (Fig. 4E), were clearly reduced as compared with those of non-tumorous mucosae.

After immunofluorescent staining, the same sections were stained with H\&E, and CRCs were confirmed using a light microscope (Fig. 4C and F).
Double immunofluorescent staining for $\beta$-catenin and Ki67 at sites of submucosal infiltration. In colon tumors from AOM/DSS mice at week 30 that showed tumor infiltration into the submucosal layer, $\beta$-catenin-positive tumor cells (green; Fig. 5A) were diffusely distributed in both, mucosal areas, and sites of submucosal infiltration, using DAPI nuclear staining (blue). In the same sections, immunofluorescent staining for Ki67 (red; Fig. 5B) was performed. Subsequently, we calculated the Ki67-positive tumor cell count/total tumor cell count separately for mucosal areas and sites of submucosal infiltration using the software. The percentage of Ki67-positive tumor cells in mucosal areas of AOM/DSS mice $(28.66 \pm 3.80 \%)$ was significantly higher than that in sites of submucosal infiltration $(10.66 \pm 1.97 \%)$ (Fig. $5 \mathrm{D}, \mathrm{n}=10$, $\mathrm{P}=0.0001)$.

After immunofluorescent staining, the same sections were stained with $\mathrm{H} \& \mathrm{E}$, and CRCs and muscularis mucosae were confirmed under a light microscope (Fig. 5C).

Immunofluorescent staining for pSmad2/3L-Thr in CRCs with submucosal infiltration and vessel invasion. pSmad2/3L-Thr-positive cells (red; arrowheads in Fig. 6) were sparsely detected around crypt bases in non-tumorous mucosae from AOM/DSS mice at week 30 (Fig. 6A), using DAPI nuclear staining (blue). In mucosal areas of colon tumors from AOM/DSS mice, pSmad2/3L-Thr-positive cells were scattered within tumor cells (open arrowhead; Fig. 6C). Furthermore, at both sites of submucosal infiltration (filled arrowheads; Fig. 6C) and vessel invasions (Fig. 6E) of these tumors, pSmad2/3L-Thr-positive cells were also detected within tumor cells.

After immunofluorescent staining, the same sections were stained with H\&E to confirm non-tumorous mucosae, CRCs, 

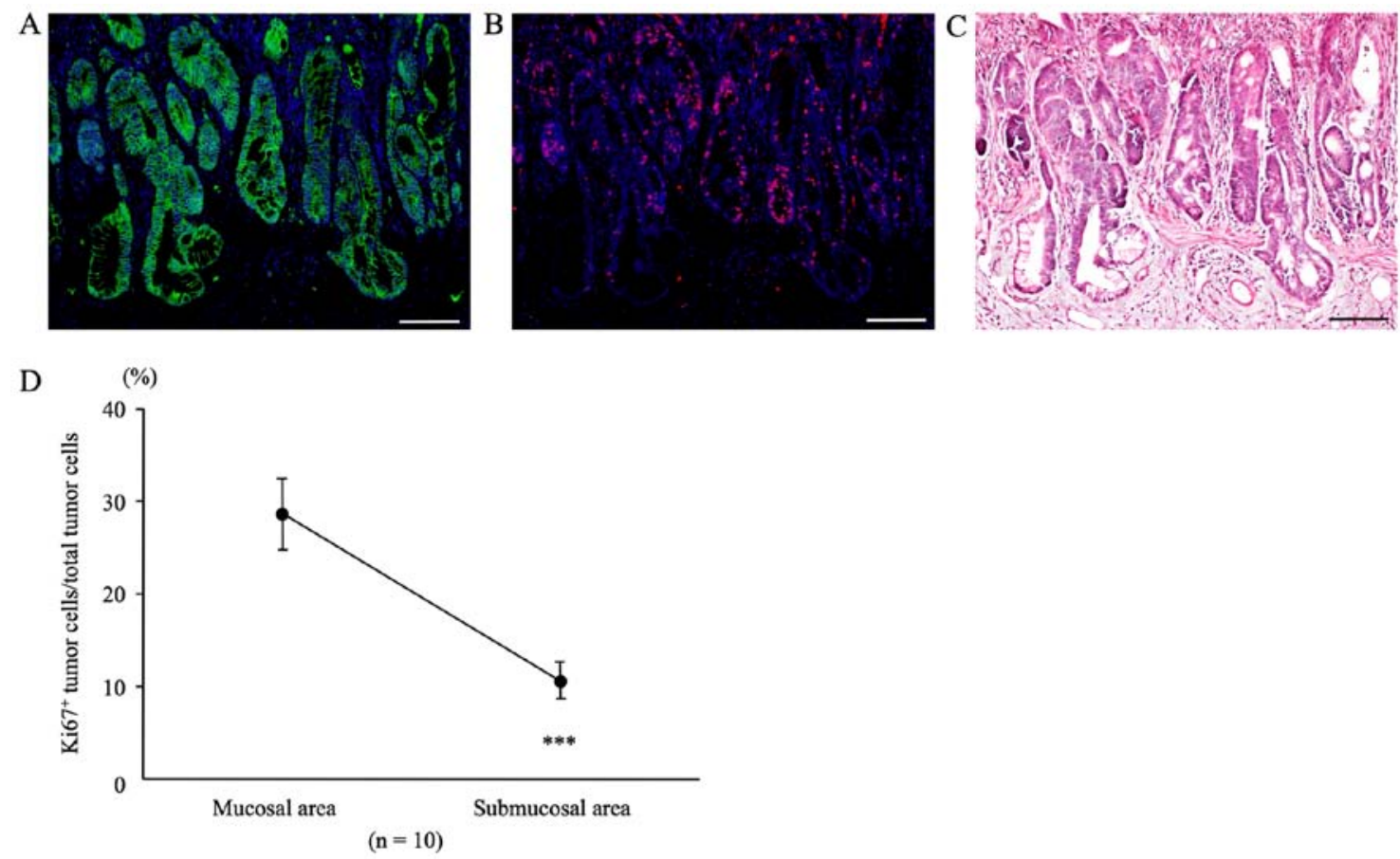

Figure 5. Double immunofluorescent staining for $\beta$-catenin (green) and Ki67 (red) in sites of submucosal tumor infiltration of AOM/DSS mice at week 30. DAPI (blue) was used for nuclear staining. (A) $\beta$-catenin-positive tumor cells were diffusely distributed in both mucosal areas and sites of submucosal infiltration in AOM/DSS mice at week 30. (B) For the same sections, immunofluorescent staining of Ki67 was performed. (C) Following immunofluorescent staining, the same sections were stained with hematoxylin and eosin, and colon tumors and muscularis mucosae were confirmed under a light microscope. Original magnification, x200. Scale bars, $100 \mu \mathrm{m}$. (D) Ki67-positive tumor cells/total tumor cells in mucosae and submucosae. The percentage of Ki67-positive tumor cells in mucosal areas of AOM/DSS mice (28.66 $\pm 3.80 \%)$ was significantly higher than that in sites of submucosal infiltration $(10.66 \pm 1.97 \% ; n=10 ; \mathrm{P}=0.0001)$. Data are presented as the mean \pm standard error of the mean, and were analyzed using a paired $\mathrm{t}$-test. ${ }^{* * *} \mathrm{P}<0.001$. AOM, azoxymethane; DSS, dextran sodium sulfate.

and pSmad2/3L-Thr-positive cells (arrowheads) under light microscopy (Fig. 6B, D and F).

Double immunofluorescent staining for pSmad2/3L-Thr and $\beta$-catenin. In colon tumors from AOM/DSS mice at week 30 that showed nuclear $\beta$-catenin expression in tumor cells, double immunofluorescent staining for pSmad2/3L-Thr (red; arrowheads in Fig. 7A) and $\beta$-catenin (green; Fig. 7B) were performed and analyzed, using DAPI nuclear staining (blue).

The percentage of pSmad2/3L-Thr-positive cells within the nuclear $\beta$-catenin-positive tumor cells $(9.98 \pm 1.82 \%)$ was significantly higher than that within the cytoplasmic $\beta$-catenin-positive tumor cells (3.67 $\pm 0.77 \%)$ (Fig. 7D, $\mathrm{n}=20, \mathrm{P}=0.0001)$.

After immunofluorescent staining, the same sections were stained with H\&E, and CRCs and pSmad2/3L-Thr-positive cells (arrowheads) were confirmed using light microscopy (Fig. 7C).

Double immunofluorescent staining for pSmad2/3L-Thr and Bmil. In colon tumors from AOM/DSS mice at week 30, double immunofluorescent staining for pSmad2/3L-Thr (red; arrowheads in Fig. 8) and Bmil (green) were performed, using DAPI nuclear staining (blue).

pSmad2/3L-Thr-positive cells were sparsely detected around crypt bases in non-tumorous mucosae from AOM/DSS mice at week 30 (Fig. 8A). In colon tumors from AOM/DSS mice, pSmad2/3L-Thr-positive cells were scattered within tumor cells (Fig. 8D). In both non-neoplastic and neoplastic epithelial cells, pSmad2/3L-Thr-positive cells showed immunohistochemical co-localization with Bmil (Fig. 8B, C, E and F).

\section{Discussion}

The prevalence of UC-associated CRCs has increased with increasing numbers of UC patients (2). The risk for developing UC-associated CRCs depends on the severity and duration of inflammation (3). The AOM/DSS mouse model manifests pathological findings represented by severe colitis with subsequent development of many colon tumors, and recapitulates the sequence of colitis-associated CRC formation in humans $(5,6)$.

Some mouse CRC models have been reported, but each has certain limitations, such as the absence of spontaneous CRC and the need for carcinogens to induce tumors. Also, in many mouse models, there are differences between animals in the development of intestinal tumors. Thus, there is an urgent need for models that more closely reflect the biology and progression of human cancer.

Mouse models of colitis-associated and sporadic CRCs, including the AOM/DSS model, show a very low incidence of invasion and metastases (8). Although chemical-induced (autochthonous) mouse CRC models have provided much information about human disease, there are still many research approaches that have not been available. In our previous study, we sacrificed AOM/DSS mice at weeks 10 or 20 , and most colon tumors were characterized by intramucosal adenocarcinoma (27). Therefore, we extended our observations until 30 weeks after AOM administration, and have developed a mouse (colitis-associated) model of advanced CRC, which shows a high degree of authenticity and consistent results for the pathology of disease progression found in human cancer patients. AOM/DSS mice, at weeks 10 and 20, showed tumor 

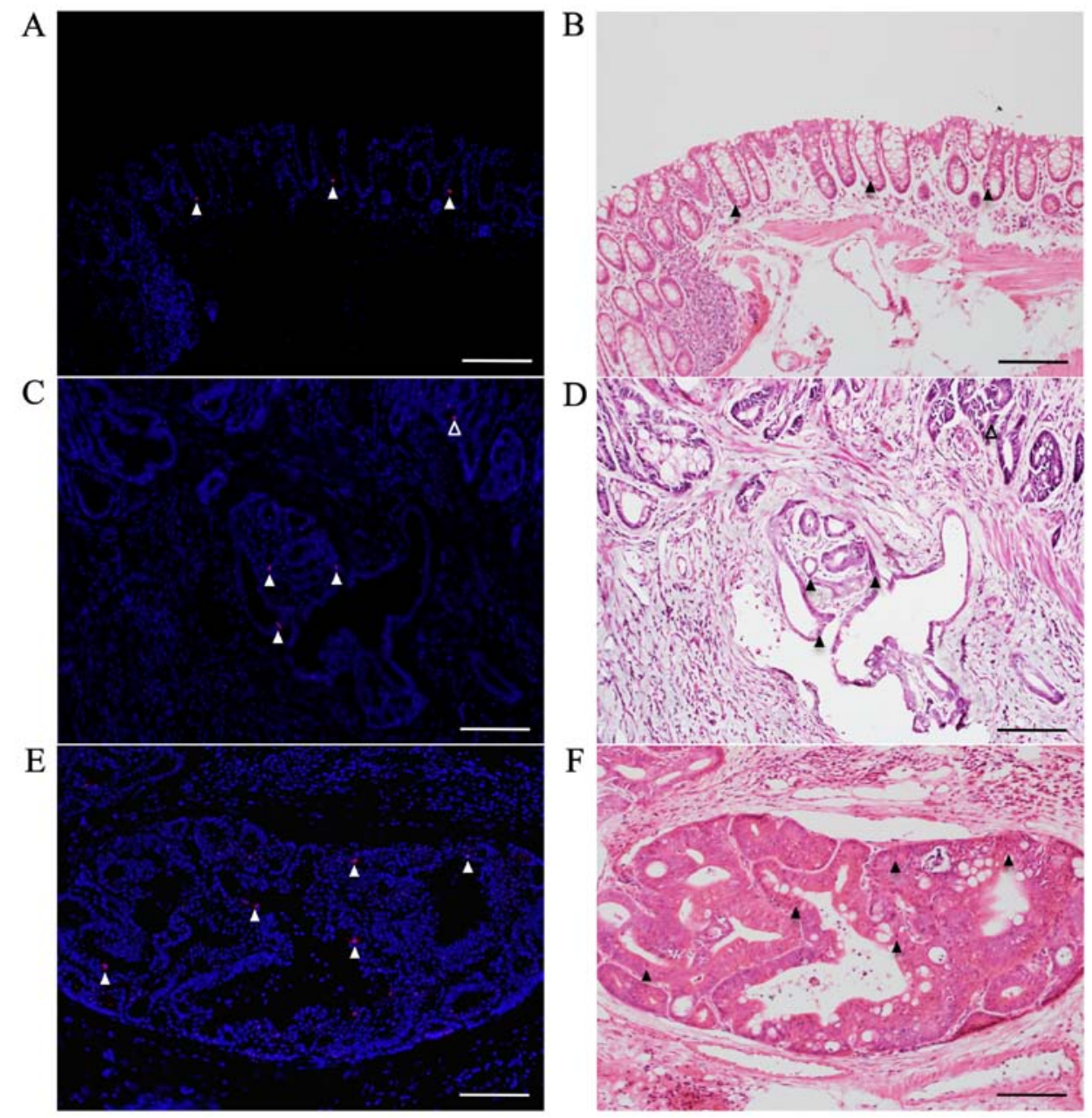

Figure 6. Immunofluorescent staining for pSmad2/3L-Thr (red; arrowheads) in non-tumorous mucosae and CRCs with submucosal infiltration and vessel invasion of AOM/DSS mice at week 30. DAPI (blue) was used for nuclear staining. (A) pSmad2/3L-Thr-positive cells were sparsely detected around crypt bases in non-tumorous mucosae. (C) In mucosal areas of colon tumors from AOM/DSS mice, pSmad2/3L-Thr-positive cells were scattered among tumor cells (open arrowhead). In both sites of (C) submucosal infiltration (filled arrowheads) and (E) vessel invasions of these tumors, pSmad2/3L-Thr-positive cells were also detected among tumor cells. (B, D and F) Following immunofluorescent staining, the same sections were stained with hematoxylin and eosin to confirm non-tumorous mucosae, colon tumors and pSmad2/3L-Thr-positive cells (arrowheads) under a light microscope. Original magnification, x200. Scale bars, $100 \mu \mathrm{m}$. AOM, azoxymethane; DSS, dextran sodium sulfate.

infiltrations into submucosa 24 and $28 \%$ of the times, respectively, whereas at week 30 it was $72 \%$. AOM/DSS mice, at weeks 10 and 20, showed tumor invasions into 0 and $4 \%$ of the vessels, respectively, whereas at week 30 the value was $40 \%$. Although no metastases to lymph nodes or other organs were observed, AOM/DSS mice were considered to acquire invasive and metastatic ability, especially between 20 and 30 weeks after AOM administration.

Recent advances in cancer biology have further emphasized the role of the immune system in creating a tumor microenvironment that promotes cancer progression through tumor growth, invasion, and metastasis. Potential uses of the model include experiments with targeted therapies and conventional drugs that affect the tumor microenvironment in the preoperative environment. Research into the role of the tumor microenvironment and the immune system, which may promote or inhibit CRC progression, is important. Therefore, mouse models used in preclinical studies must have complete immune responses to reveal more detailed interactions with human cancer. The tumor microenvironment is particularly important in the context of metastatic disease, where new therapies are still in great need. Because drugs aimed at regulating these cancer progression factors are developed and evaluated preclinically, model systems in which tumors have advanced will play major roles. In the future, if our method can be improved in AOM/DSS mice to establish a CRC model with metastasis to lymph nodes and organs, it is expected to provide a mouse metastatic CRC model that is particularly useful in preclinical studies for the development of drugs that target those mechanisms.

The proteins involved in the regulation of cytoskeletal apparatus function in conjunction with the junctional proteins such as $\beta$-catenin and E-cadherin. Their dynamics influence functional integrity that affects cell migration, invasion, and polarity. $\beta$-catenin is a pivotal component of the E-cadherin-mediated cell-cell adhesion system, and a key molecule in the Wnt-APC signaling pathway (34). It controls the transcription of genes involved in cell growth, development, and differentiation. $\beta$-catenin is found in the cell membrane of non-tumorous colon epithelial cells, but nuclear and cytoplasmic $\beta$-catenin accumulation is associated with carcinogenesis in the colon (35). Consistent with earlier 

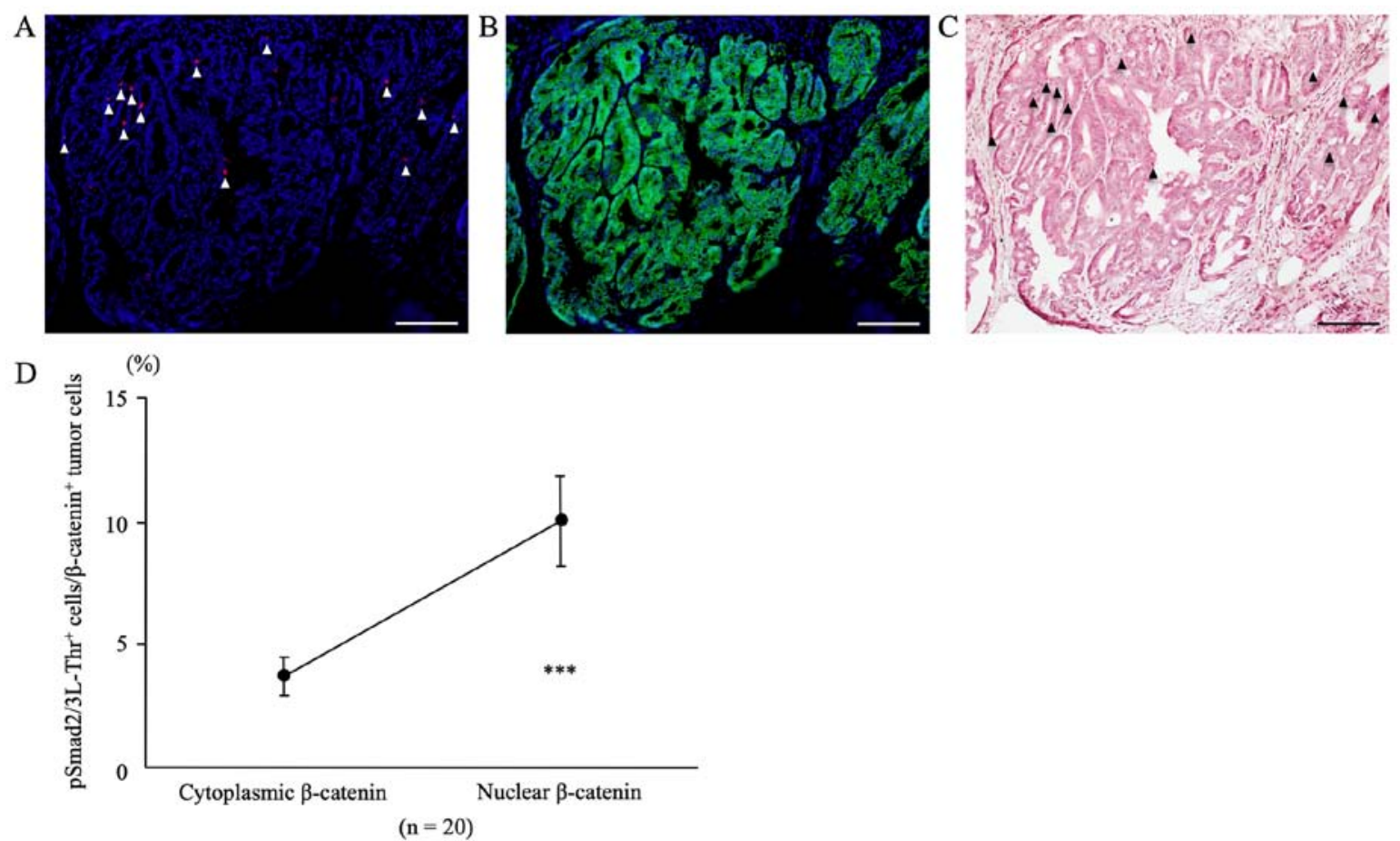

Figure 7. Double immunofluorescent staining for $\mathrm{pSmad} 2 / 3 \mathrm{~L}-\mathrm{Thr}$ (red; arrowheads) and $\beta$-catenin (green) in colon tumors from azoxymethane/dextran sodium sulfate mice at week 30. DAPI (blue) was used for nuclear staining. (A) pSmad2/3L-Thr-positive cells (arrowhead) were scattered among tumor cells. (B) For the same sections, immunofluorescent staining of $\beta$-catenin revealed cytoplasmic and nuclear $\beta$-catenin expression in tumor cells. (C) Following immunofluorescent staining, the same sections were stained with hematoxylin and eosin, and colon tumors and pSmad2/3L-Thr-positive cells (arrowheads) were confirmed by light microscopy. Original magnification, x200. Scale bars, $100 \mu \mathrm{m}$. (D) pSmad2/3L-Thr-positive cells/ $\beta$-catenin-positive tumor cells within cytoplasm and nuclei. The percentage of pSmad2/3L-Thr-positive cells among the nuclear $\beta$-catenin-positive tumor cells $(9.98 \pm 1.82 \%)$ was significantly higher than that among the cytoplasmic $\beta$-catenin-positive tumor cells $(3.67 \pm 0.77 \% ; \mathrm{n}=20 ; \mathrm{P}=0.0001)$. Data are presented as the mean \pm standard error of the mean, and were analyzed using a paired t-test. ${ }^{* * *} \mathrm{P}<0.001$.
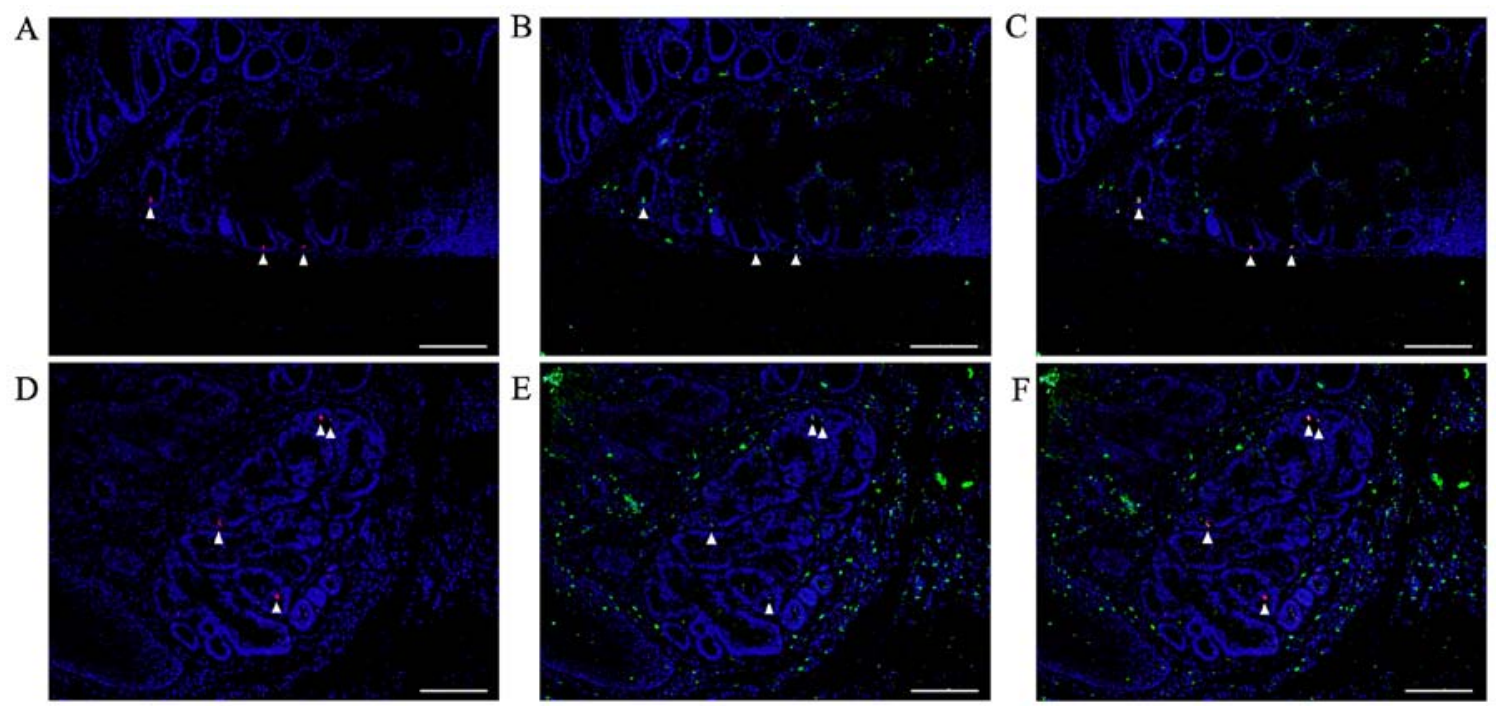

Figure 8. Double immunofluorescent staining for pSmad2/3L-Thr (red; arrowheads) and Bmi1 (green) in non-tumorous mucosae and CRCs of AOM/DSS mice at week 30. DAPI (blue) was used for nuclear staining. (A) pSmad2/3L-Thr-positive cells were sparsely detected around crypt bases in non-tumorous mucosae. (D) In colon tumors from AOM/DSS mice, pSmad2/3L-Thr-positive cells were scattered among tumor cells. pSmad2/3L-Thr-positive cells exhibited immunohistochemical co-localization with Bmil in both (B and C) non-neoplastic and (E and F) neoplastic epithelial cells, (C and F) as indicated in the merged panels. Original magnification, x200. Scale bars, $100 \mu \mathrm{m}$. AOM, azoxymethane; DSS, dextran sodium sulfate.

studies, we detected the nuclear and cytoplasmic $\beta$-catenin accumulation in colon tumors of AOM/DSS mice, including vessel invasions. Many studies have established the link between loss of E-cadherin expression in cancer cells and transition to EMT $(36,37)$. Expression levels of E-cadherin differ dramatically among human tumors, demonstrating that there is a positive correlation between E-cadherin levels and patient survival (38). In this regard, mutations in the E-cadherin gene have been identified in cancer cells and are thought to facilitate EMT development and cancer cell metastasis $(39,40)$. 
Consistent with these studies, we detected strong E-cadherin positivity in the cell membrane of the parts of non-tumorous mucosae in AOM/DSS mice, and levels of E-cadherin positivity in the cell membrane were clearly attenuated in the parts of colon tumors, especially in AOM/DSS mice at week 30, suggesting strong induction and promotion of EMT.

$\mathrm{Ki} 67$ antigen is a nuclear matrix protein expressed in proliferating cells, but not in quiescent cells (41). In colon tumors from AOM/DSS mice at week 30 that showed tumor infiltration into the submucosal layer, the percentage of Ki67-positive tumor cells at the sites of submucosal infiltration, the invasion front of CRCs, was significantly lower than that in mucosal areas. This finding is consistent with previous reports that the invasion front of human CRCs shows low proliferative activity (42-44).

In our previous studies, we have confirmed significant expression of $\mathrm{pSmad} 2 / 3 \mathrm{~L}-\mathrm{Thr}$ in normal colon epithelial cells of wild-type mice, and in tumorous colon epithelial cells of AOM/DSS mice, suggesting that these cells are colon epithelial stem-like cells and colorectal CSCs, respectively $(26,27)$. In addition, we have shown that pSmad2/3L-Thr-positive cells are BrdU label-retaining, slow-cycling, and Ki67-negative quiescent cells in the G0 phase, located adjacent to actively proliferating cells of normal esophageal and colon epithelial cells $(26,45)$. We have consistently advocated that pSmad2/3L-Thr helps identify normal epithelial stem-like cells in the esophagus, stomach, small intestine, and colon and colorectal CSCs, immediately before they re-enter the cell cycle from the dormant state of the G0 phase $(26,27,33,45)$. Moreover, in the present study, pSmad2/3L-Thr-positive cells were detected in both, the sites of submucosal infiltration as well as vessel invasion of tumors in AOM/DSS mice at week 30. Additionally, the percentage of $\mathrm{pSmad} 2 / 3 \mathrm{~L}-\mathrm{Thr}$-positive cells within the nuclear $\beta$-catenin-positive tumor cells was significantly higher than that within the cytoplasmic $\beta$-catenin-positive tumor cells. This result is consistent with previous reports suggesting that nuclear $\beta$-catenin accumulation is important for regulating intranuclear CSC-related transcription factors and maintaining the CSC phenotype $(46,47)$. We performed double immunofluorescent staining for pSmad2/3L-Thr and Bmi1, which is a representative marker of slow-cycling (cancer) stem cells $(48,49)$. pSmad2/3L-Thr-positive cells showed immunohistochemical co-localization with Bmil in non-tumorous mucosae and tumors in AOM/DSS mice at week 30 . We were able to re-confirm the results supporting that pSmad2/3L-Thr is a biomarker of tissue and cancer stem cells.

CSC theory suggests that a small number of undifferentiated cancer cells that exhibit normal stem cell-like characteristics promote tumor growth and spread. Although CSCs are capable of self-renewal, they are relatively dormant and are capable of proliferation, although not often cycling. They have been shown to have significantly longer cell-cycle times when compared to proliferating non-CSCs. It is presumed that this is due to the arrest of CSCs in the G0 phase (50). Although CRC has been thoroughly studied, little is known about the original cells of carcinogenesis. In both non-neoplastic and neoplastic epithelial cells, pSmad2/3L-Thr-positive cells consistently exhibit stem-like properties. pSmad2/3L-Thr has a high probability of a stem-like cell biomarker in other organs and CSCs. Future studies are needed to further confirm that pSmad2/3L-Thr-positive cells are CSCs in other neoplastic lesions.
In conclusion, in this study, we developed a mouse (colitis-associated) advanced CRC model that showed tumor infiltration into the submucosa and invasion into the vessels, with the results of this study compelling us to re-acknowledge the theory that pSmad2/3L-Thr immunostaining-positive cells are CSCs.

\section{Acknowledgements}

Not applicable.

\section{Funding}

The present study was supported by a Grant-in-Aid for Scientific Research (C) (grant nos. 16K09330 and 25460938) from the Japan Society for the Promotion of Science.

\section{Availability of data and materials}

The datasets used and/or analyzed during the current study are available from the corresponding author on reasonable request.

\section{Authors' contributions}

All authors have contributed to and agreed on the content of the manuscript. YT carried out the experiments, conducted data analyses and drafted the manuscript. TF conceived the study, carried out data analyses, performed the statistical analyses and helped to draft the manuscript. SH, YM, SM, RS, TTa, TTo, TI, YA, AN and KO performed data analyses, and provided significant advice and consultation. All authors read and approved the final manuscript.

\section{Ethics approval and consent to participate}

All experimental protocols were approved by the Ethics Committee for the Use of Experimental Animals of Kansai Medical University (approval no. 19-006).

\section{Patient consent for publication}

Not applicable.

\section{Competing interests}

The authors declare that they have no competing interests.

\section{References}

1. Eaden JA, Abrams KR and Mayberry JF: The risk of colorectal cancer in ulcerative colitis: A meta-analysis. Gut 48: 526-535, 2001.

2. Ekbom A, Helmick C, Zack M and Adami HO: Ulcerative colitis and colorectal cancer. A population-based study. N Engl J Med 323: 1228-1233, 1990.

3. Seril DN, Liao J, Yang GY and Yang CS: Oxidative stress and ulcerative colitis-associated carcinogenesis: Studies in humans and animal models. Carcinogenesis 24: 353-362, 2003.

4. Tanaka T: Development of an inflammation-associated colorectal cancer model and its application for research on carcinogenesis and chemoprevention. Int J Inflamm 2012: 658786, 2012.

5. Tanaka T, Kohno H, Suzuki R, Yamada Y, Sugie S and Mori H: A novel inflammation-related mouse colon carcinogenesis model induced by azoxymethane and dextran sodium sulfate. Cancer Sci 94: 965-973, 2003. 
6. Tanaka T: Colorectal carcinogenesis: Review of human and experimental animal studies. J Carcinog 8: 5, 2009.

7. Boivin GP, Washington K, Yang K, Ward JM, Pretlow TP, Russell R, Besselsen DG, Godfrey VL, Doetschman T, Dove WF, et al: Pathology of mouse models of intestinal cancer: Consensus report and recommendations. Gastroenterology 124: 762-777, 2003.

8. Rosenberg DW, Giardina C and Tanaka T: Mouse models for the study of colon carcinogenesis. Carcinogenesis 30: 183-196, 2009

9. Mani SA, Guo W, Liao MJ, Eaton EN, Ayyanan A, Zhou AY, Brooks M, Reinhard F, Zhang CC, Shipitsin M, et al: The epithelial-mesenchymal transition generates cells with properties of stem cells. Cell 133: 704-715, 2008.

10. Kalluri R and Weinberg RA: The basics of epithelial-mesenchymal transition. J Clin Invest 119: 1420-1428, 2009.

11. Clarke MF, Dick JE, Dirks PB, Eaves CJ, Jamieson $\mathrm{CH}$, Jones DL, Visvader J, Weissman IL and Wahl GM: Cancer stem cells - perspectives on current status and future directions: AACR Workshop on cancer stem cells. Cancer Res 66: 9339-9344, 2006.

12. Campbell LL and Polyak K: Breast tumor heterogeneity: Cancer stem cells or clonal evolution? Cell Cycle 6: 2332-2338, 2007.

13. Sarkar FH, Li Y, Wang Z and Kong D: Pancreatic cancer stem cells and EMT in drug resistance and metastasis. Minerva Chir 64: 489-500, 2009.

14. Brabletz T, Hlubek F, Spaderna S, Schmalhofer O Hiendlmeyer E, Jung A and Kirchner T: Invasion and metastasis in colorectal cancer: Epithelial-mesenchymal transition, mesenchymal-epithelial transition, stem cells and beta-catenin. Cells Tissues Organs 179: 56-65, 2005.

15. Heldin $\mathrm{CH}$, Miyazono K and ten Dijke P: TGF-beta signalling from cell membrane to nucleus through SMAD proteins Nature 390: 465-471, 1997.

16. Massagué J: TGF-beta signal transduction. Annu Rev Biochem 67 753-791, 1998

17. Wrana JL: Crossing Smads. Sci STKE 2000: re1, 2000.

18. Kretzschmar M, Doody J, Timokhina I and Massagué J: A mechanism of repression of TGFbeta/Smad signaling by oncogenic Ras. Genes Dev 13: 804-816, 1999.

19. Matsuura I, Denissova NG, Wang G, He D, Long J and Liu F: Cyclin-dependent kinases regulate the antiproliferative function of Smads. Nature 430: 226-231, 2004.

20. Mori S, Matsuzaki K, Yoshida K, Furukawa F, Tahashi Y, Yamagata H, Sekimoto G, Seki T, Matsui H, Nishizawa M, et al TGF-beta and HGF transmit the signals through JNK-dependent Smad2/3 phosphorylation at the linker regions. Oncogene 23 : 7416-7429, 2004.

21. Tarasewicz E and Jeruss JS: Phospho-specific Smad3 signaling: Impact on breast oncogenesis. Cell Cycle 11: 2443-2451, 2012

22. Matsuzaki K: Smad3 phosphoisoform-mediated signaling during sporadic human colorectal carcinogenesis. Histol Histopathol 21 : 645-662, 2006

23. Matsuzaki K, Kitano C, Murata M, Sekimoto G, Yoshida K Uemura Y, Seki T, Taketani S, Fujisawa J and Okazaki K: Smad2 and Smad3 phosphorylated at both linker and $\mathrm{COOH}$-terminal regions transmit malignant TGF-beta signal in later stages of human colorectal cancer. Cancer Res 69: 5321-5330, 2009.

24. Sapkota G, Knockaert M, Alarcón C, Montalvo E, Brivanlou AH and Massagué J: Dephosphorylation of the linker regions of Smad1 and $\mathrm{Smad} 2 / 3$ by small C-terminal domain phosphatases has distinct outcomes for bone morphogenetic protein and transforming growth factor-beta pathways. J Biol Chem 281: 40412-40419, 2006.

25. Derynck $R$ and Zhang YE: Smad-dependent and Smad-independent pathways in TGF-beta family signalling. Nature 425: 577-584, 2003

26. Kishimoto M, Fukui T, Suzuki R, Takahashi Y, Sumimoto K Okazaki T, Sakao M, Sakaguchi Y, Yoshida K, Uchida K, et al: Phosphorylation of Smad2/3 at specific linker threonine indicates slow-cycling intestinal stem-like cells before reentry to cell cycle. Dig Dis Sci 60: 362-374, 2015.

27. Suzuki R, Fukui T, Kishimoto M, Miyamoto S, Takahashi Y Takeo M, Mitsuyama T, Sakaguchi Y, Uchida K, Nishio A, et al: Smad2/3 linker phosphorylation is a possible marker of cancer stem cells and correlates with carcinogenesis in a mouse model of colitis-associated colorectal cancer. J Crohn's Colitis 9: $565-574,2015$

28. Suzuki R, Kohno H, Sugie S and Tanaka T: Sequential observations on the occurrence of preneoplastic and neoplastic lesions in mouse colon treated with azoxymethane and dextran sodium sulfate. Cancer Sci 95: 721-727, 2004.

29. Ward JM: Morphogenesis of chemically induced neoplasms of the colon and small intestine in rats. Lab Invest 30: 505-513, 1974
30. Murata M, Matsuzaki K, Yoshida K, Sekimoto G, Tahashi Y, Mori S, Uemura Y, Sakaida N, Fujisawa J, Seki T, et al: Hepatitis B virus $X$ protein shifts human hepatic transforming growth factor (TGF)-beta signaling from tumor suppression to oncogenesis in early chronic hepatitis B. Hepatology 49: 1203-1217, 2009.

31. Sekimoto G, Matsuzaki K, Yoshida K, Mori S, Murata M, Seki T, Matsui H, Fujisawa J and Okazaki K: Reversible Smad-dependent signaling between tumor suppression and oncogenesis. Cancer Res 67: 5090-5096, 2007.

32. Furukawa F, Matsuzaki K, Mori S, Tahashi Y, Yoshida K, Sugano Y, Yamagata H, Matsushita M, Seki T, Inagaki Y, et al: p38 MAPK mediates fibrogenic signal through Smad3 phosphorylation in rat myofibroblasts. Hepatology 38: 879-889, 2003.

33. Fukui T, Kishimoto M, Nakajima A, Yamashina M, Nakayama S, Kusuda T, Sakaguchi Y, Yoshida K, Uchida K, Nishio A, et al: The specific linker phosphorylation of Smad2/3 indicates epithelial stem cells in stomach; particularly increasing in mucosae of Helicobacter-associated gastritis. J Gastroenterol 46: 456-468, 2011.

34. Miller JR and Moon RT: Signal transduction through beta-catenin and specification of cell fate during embryogenesis. Genes Dev 10: 2527-2539, 1996

35. Brabletz T, Jung A and Kirchner T: Beta-catenin and the morphogenesis of colorectal cancer. Virchows Arch 441: 1-11, 2002.

36. Edelman GM, Gallin WJ, Delouvée A, Cunningham BA and Thiery JP: Early epochal maps of two different cell adhesion molecules. Proc Natl Acad Sci USA 80: 4384-4388, 1983.

37. Tepass U, Truong K, Godt D, Ikura M and Peifer M: Cadherins in embryonic and neural morphogenesis. Nat Rev Mol Cell Biol 1: 91-100, 2000

38. Hirohashi S: Inactivation of the E-cadherin-mediated cell adhesion system in human cancers. Am J Pathol 153: 333-339, 1998.

39. Muta H, Noguchi M, Kanai Y, Ochiai A, Nawata H and Hirohashi S: E-cadherin gene mutations in signet ring cell carcinoma of the stomach. Jpn J Cancer Res 87: 843-848, 1996.

40. Saito A, Kanai Y, Maesawa C, Ochiai A, Torii A and Hirohashi S: Disruption of E-cadherin-mediated cell adhesion systems in gastric cancers in young patients. Jpn J Cancer Res 90: 993-999, 1999.

41. Weidner N, Moore DH II and Vartanian R: Correlation of Ki-67 antigen expression with mitotic figure index and tumor grade in breast carcinomas using the novel 'paraffin'-reactive MIB1 antibody. Hum Pathol 25: 337-342, 1994.

42. Jung A, Schrauder M, Oswald U, Knoll C, Sellberg P,Palmqvist R, Niedobitek G, Brabletz T and Kirchner T: The invasion front of human colorectal adenocarcinomas shows co-localization of nuclear beta-catenin, cyclin D1, and p16INK4A and is a region of low proliferation. Am J Pathol 159: 1613-1617, 2001.

43. Palmqvist R, Rutegârd JN, Bozoky B, Landberg G and Stenling R: Human colorectal cancers with an intact p16/cyclin D1/pRb pathway have up-regulated p16 expression and decreased proliferation in small invasive tumor clusters. Am J Pathol 157: 1947-1953, 2000

44. Palmqvist R, Sellberg $\mathrm{P}$, Oberg A, Tavelin B, Rutegård JN and Stenling R: Low tumour cell proliferation at the invasive margin is associated with a poor prognosis in Dukes' stage B colorectal cancers. Br J Cancer 79: 577-581, 1999.

45. Takahashi Y, Fukui T, Kishimoto M, Suzuki R, Mitsuyama T, Sumimoto K, Okazaki T, Sakao M, Sakaguchi Y, Yoshida K, et al: Phosphorylation of Smad2/3 at the specific linker threonine residue indicates slow-cycling esophageal stem-like cells before re-entry to the cell cycle. Dis Esophagus 29: 107-115, 2016.

46. Li XQ, Yang XL, Zhang G, Wu SP, Deng XB, Xiao SJ, Liu QZ, Yao KT and Xiao GH: Nuclear $\beta$-catenin accumulation is associated with increased expression of Nanog protein and predicts poor prognosis of non-small cell lung cancer. J Transl Med 11: 114,2013 .

47. Zhang G, Wang W, Yao C, Zhang S, Liang L, Han M, Ren J, Qi X, Zhang X, Wang S, et al: Radiation-resistant cancer stem-like cell properties are regulated by PTEN through the activity of nuclear $\beta$-catenin in nasopharyngeal carcinoma. Oncotarget 8 : 74661-74672, 2017.

48. Espersen ML, Olsen J, Linnemann D, Høgdall E and Troelsen JT: Clinical implications of intestinal stem cell markers in colorectal cancer. Clin Colorectal Cancer 14: 63-71, 2015.

49. Srinivasan T, Walters J, Bu P, Than EB, Tung KL, Chen KY, Panarelli N, Milsom J, Augenlicht L, Lipkin SM, et al: NOTCH signaling regulates asymmetric cell fate of fast- and slow-cycling colon cancer-initiating cells. Cancer Res 76: 3411-3421, 2016.

50. Boman BM, Fields JZ, Cavanaugh KL, Guetter A and Runquist OA: How dysregulated colonic crypt dynamics cause stem cell overpopulation and initiate colon cancer. Cancer Res 68: 3304-3313, 2008. 